\section{Nicholas Ayache}

INRIA - The French National Institute for Research in Computer Science and Control Sophia-Antipolis, France

\title{
Preface
}

\section{From Digital Anatomy to Virtual Scalpels and Image Guided Therapy}

Medical imaging informatics will bring about a revolution in medicine in the 21stcentury, introducing a collection of powerful new tools designed to better assist clinical diagnosis and to model, simulate, and guide patient's therapy more efficiently. A new discipline is emerging in computer science, closely related to others such as computer vision, computer graphics, artificial intelligence and robotics.

How could a plausible scenario of tomorrow's hospital room look? First, all imaging modalities will be interconnected to fuse the available anatomical and functional data of a patient and exploit them in a coherent manner. The images involved will not only be radiographs, computed tomographies, magnetic resonance, nuclear medicine, or ultrasound images, but also video, endoscopic, confocal, microscopic, histological, or molecular images. This information will be processed in 3-D, as most imaging modalities are volumetric, but also in 4-D whenever temporal sequences are available, either to detect subtle changes or to quantify a dynamic motion (typically for cardiac analysis). The physician will, therefore, be provided with new sets of quantitative measurements to assess a more accurate and objective diagnosis. At this stage, digital image processing will often play the same role as the microscope during the 17th century, allowing visualization of details or changes which otherwise, would have remained invisible, by a direct visualization of the original images $(*)$.

When a therapy has been decided upon, pre-operative diagnostic images will serve tooptimally prepare a therapy. Mostly interactive, the software tools will enhance the capacities of the physicians, offering the possibility to experiment various approaches before selecting the best one. Virtual reality display and force feed-back (haptic) devices will provide realistic training for therapy, allowing the surgeon to practice on virtual but highly realistic models and scalpels. Once the actual treatment has to be delivered, imageguided therapy procedures will rely on various types of intra-operative images. Augmented reality tools will enhance the perceptual capacities of the physician by fusing the intra-operative images with pre-operative images and models, including, for instance, high resolution digital atlases.

High speed networks will permit the transmission of images and gestures to remote sites, where medical robots will enhance, or replace, the physicians' gestures, often with help of real-time processing of intra-operative images. 
This will allow, for instance, compensation of a moving organ (e.g. the beating heart), offering the possibility for surgeons to operate on a quasi-still image while a robot adds the required computed motion to his gestures. Finally, post-operativeimages will be automatically compared to preoperative images, in order to better assess the efficiency of the therapy. This will also be useful to objectively measure the efficiency of a drug treatment and, thus, better control the design of new medication and therapeutic protocols.

Is this scenario a dream? Certainly not. We know that parts of this scenario are already operational in a few leading medical centers. Also, research on these topics is more active than ever across the globe, in all areas of medical image processing and analysis, including the acquisition, reconstruction, enhancement, compression, storage, transmission, visualization and understanding of medical images. The major methodological research topics that have emerged include segmentation, registration and fusion, dynamic analysis from temporal sequences, modeling of anatomy and physiology from anatomical or functional images, content-based indexing, statistical analysis of shapes and signals, surgery simulation and image-guided medical robotics. These topics often involve interdisciplinary methodologies. For instance, to create a digital model whose behavior mimics the actual body of a given patient as well as possible, surgery simulation requires geometric anatomical modeling with various modalities of medical imaging, biomechanical modeling of soft tissues, and the physiological modeling of organs and vessels.

A leading selection of articles in this Yearbook describe the state of the art developments of some of the most advanced research centers in medical informatics, and suggest some contours of this upcoming revolution.

(*) Citation from Gilles Kahn, Scientific Director of INRIA.

\section{References}

1. Bankman I, editor. Handbook of Medical Imaging - Processing and Analysis, Academic Press; 2000.

2. CAS: Int. Journal of Computer Aided Surgery, Wiley, http://www3.interscience. wiley.com/cgi-bin/jtoc?ID=56867

3. Duncan J, Ayache N. Medical Image Analysis: Progress over two decades and the challenges ahead. IEEE Transactions on Pattern Analysis and Machine Intelligence
2000;22(1):85-106.

4. Grimson E, Kikinis R, Jolesz F, Black P, Image Guided Surgery, Scientific American, June 1999.

5. Marescaux J, Clement JM, Tassetti V, Koehl C, Cotin S, Russier Y, et al. Virtual Reality Applied to Hepatic Surgery Simulation: The Next Revolution. Ann Surg 1998 Nov;228(5):627-34.

6. MedIA: Int. Journal on Medical Image Analysis, Elsevier. http://www.elsevier.nl/ locate/media

7. Medinfo'01: 10th World Congress on Health and Medical Informatics; http:// www.medinfo2001.org/

1. MICCAI'01: 4th Int. Conf. on Medical Image Computing and Computer-Assisted Intervention Conference, Utrecht, Holland, Oct. 2001; http://miccai.isi.uu.nl/

8. Robb RA. Biomedical Imaging, Visualization and Analysis. New York, NY: John Wiley and Sons, Inc.; 1999.

6. Taylor RH, Lavallee S, Burdea G, Mosges $\mathrm{R}$, editors. Computer-Integrated Surgery. Cambridge, Massachusetts: MIT Press; 1996.

8. TMI: IEEE Transactions on Medical Imaging,http://ieeexplore.ieee.org/lpdocs/ epic03/RecentIssues.htm?punumber $=42$

Address of the author:

Nicholas Ayache

Research Director, Epidaure Project,

Medical Imaging and Robotics

INRIA Sophia Antipolis

2004 Route des Lucioles

F-06902 Sophia-Antipolis, France

E-mail: nicholas.ayache@sophia.inria.fr

URL: http://www.inria.fr/personnel/

Nicholas.Ayache 